\title{
Risco e Proteção: Em Busca de Um Equilíbrio Promotor de Resiliência ${ }^{1}$
}

\author{
Renata P. Pesce ${ }^{2}$ \\ Simone G. Assis \\ Nilton Santos \\ Raquel de V. Carvalhaes de Oliveira \\ Fundação Oswaldo Cruz
}

\begin{abstract}
RESUMO - O artigo analisa a relação da resiliência com eventos de vida desfavoráveis e fatores de proteção. A amostra do estudo foi de 997 adolescentes escolares da rede pública de ensino de São Gonçalo/RJ. Como medida de resiliência utilizouse a Escala de Resiliência desenvolvida por Wagnild e Young (1993). Para medir eventos de vida foram utilizadas escalas de violência física (Straus, 1979) e psicológica (Pitzner \& Drummond, 1997), itens de violência na escola e na localidade, violência entre irmãos e entre pais, e violência sexual entre outros. Como fatores de proteção utilizou-se: Escala de Apoio Social de Shebourne e Stewart (conforme citado por Chor, Grip, Lopes \& Farstein, 2001), Escala de Auto-Estima (Rosemberg, 1989), itens abordando supervisão familiar, relacionamento com amigos e professores. Os eventos de vida negativos não apresentaram relação com a resiliência, enquanto os fatores de proteção mostraram-se todos correlacionados com o constructo.
\end{abstract}

Palavras chave: eventos de vida; fatores de proteção; resiliência.

\section{Risk and Protection: Looking for an Equilibrium That Provides Resilience}

\begin{abstract}
The article analyzes the relationship of the resilience with unfavorable life events and protection factors. The sample of that study belonged to 997 school adolescents of the public schools of São Gonçalo/RJ. As measure of resilience was used the Resilience Scale developed by Wagnild \& Young (1993). To measure Life Events were used scale of physical violence (Straus, 1979) and psychological violence (Pitzer \& Drummond, 1997), violence items in the school and in the place, violence among siblings and among parents, sexual violence and others. As protection factors were used the Scale of Social Support of Shebourne e Stewart (Chor, Grip, Lopes \& Farstein, 2001), the Scale of Self-Esteem of Rosemberg (1989), family supervision, relationship with friends and teachers. It was observed that the negative life events didn't present relationship with the resilience. On the other hand, the appraised protection factors were shown all correlated with the resilience.
\end{abstract}

Key words: life events; protection factors; resilience.

Compreende-se resiliência como o conjunto de processos sociais e intrapsíquicos que possibilitam o desenvolvimento de uma vida sadia, mesmo vivendo em um ambiente não sadio. Este processo resulta da combinação entre os atributos da criança ou jovem e seu ambiente familiar, social e cultural. Deste modo, a resiliência não pode ser pensada como um atributo que nasce com o sujeito, nem que ele adquire durante seu desenvolvimento. É um processo interativo entre a pessoa e seu meio, considerado como uma variação individual em resposta ao risco, sendo que os mesmos fatores causadores de estresse podem ser experenciados de formas diferentes por pessoas diferentes, não sendo a resiliência um atributo fixo do indivíduo (Rutter, 1987).

Junqueira e Deslandes (2003), a partir de uma revisão crítica das publicações sobre resiliência do final dos anos 1990 , verificaram que o conceito não apresenta uma definição consensual, sendo caracterizado em termos mais

1 Agradecemos o apoio do UNICEF, FAPERJ e ao PIBIC (CNPq).

2 Endereço: CLAVES - Centro Latino Americano de Estudos de Violência e Saúde/ FIOCRUZ, Av. Brasil, 4.036 sala 700, Manguinhos, RJ, Brasil 21040-361. E-mail: rpesce@ claves.fiocruz.br operacionais do que descritivo. As autoras consideram a resiliência como a capacidade do sujeito de, em determinados momentos e de acordo com as circunstâncias, lidar com a adversidade não sucumbindo a ela, alertando para a necessidade de relativizar, em função do indivíduo e do contexto, o aspecto de "superação" de eventos potencialmente estressores apontado em algumas definições de resiliência. Defendem que o termo resiliência traduz conceitualmente a possibilidade de superação num sentido dialético, o que representa não uma eliminação, mas uma re-significação do problema.

\section{Eventos de vida adversos como fatores de risco}

São muitos os fatores de risco, crônicos ou agudos, que estariam afetando a capacidade de resiliência de crianças e adolescentes. Condições de pobreza, rupturas na família, vivência de algum tipo de violência, experiências de doença no próprio indivíduo ou na família e perdas importantes são alguns exemplos.

Eventos considerados como risco são obstáculos individuais ou ambientais que aumentariam a vulnerabilidade da 
criança para resultados negativos no seu desenvolvimento. Embora a análise do risco inicialmente tenha examinado esses fatores como eventos estáticos, eventualmente, análises mais sofisticadas sugerem que o risco é um processo, e que, por exemplo, o número total de fatores de risco a que uma criança foi exposta, o período de tempo, o momento da exposição ao risco e o contexto são mais importantes do que uma única exposição grave (Engle, Castle \& Menon, 1996).

Considerando-se que as experiências de vida negativas são inevitáveis para qualquer indivíduo, sobressai a questão dos níveis de exposição e dos limites individuais de cada um. Assim, a visão subjetiva de um indivíduo a determinada situação, ou seja, sua percepção, interpretação e sentido atribuído ao evento estressor é que o classificará ou não como condição de estresse. Por essa razão, um evento pode ser enfrentado como perigo por um indivíduo e para outro, ser apenas um desafio (Yunes \& Szymanski, 2001).

Não há também consenso quanto ao número de eventos de vida negativos necessários para afetar a capacidade de resiliência do indivíduo. A hipótese de que um indivíduo poderia sofrer apenas um evento de risco sucumbiria perante a interseção existente entre os fenômenos na vida cotidiana: o divórcio dos pais, a transição para a puberdade ou gravidez na adolescência geralmente trazem consigo uma constelação de outros eventos associados (Gore \& Eckenrode, 1996).

Outra discussão refere-se ao fato de que o acúmulo de fatores de risco poderia predizer a resiliência, já que todo ser humano possui um limite para lidar com o estresse. Celia e Souza (2002) comentam que ter mais do que quatro eventos estressantes na vida eleva a condição de risco. Outros autores advertem para o perigo da utilização do somatório de itens de eventos de vida encontrados em escalas e instrumentos para definir um indivíduo de alto risco. É preciso considerar a heterogeneidade dos tipos de eventos que muitas vezes são classificados da mesma forma, além do que, muitas vezes o mais importante não é apenas saber se o respondente vivenciou determinada situação, mas também como o evento afetou o indivíduo (Garmezy, 1996; Luthar \& Cushing, 1999; Luthar \& Zigler, 1991).

Garmezy (1988) sugere que eventos agudos podem ser infinitamente mais desastrosos do que condições crônicas a que o indivíduo já está habituado. Os eventos envolvendo perdas e separações de pessoas queridas são exemplos desse tipo. Anos depois, esse mesmo autor admitiu que o poder do acúmulo de eventos e a cronicidade da adversidade tem se tornado evidente nos estudos de risco e que essa abordagem vem substituir o foco anteriormente dado a eventos específicos como antecedentes às desordens de comportamento. As situações de pobreza, segundo o autor, são campos favoráveis para essa investigação pois, nessas condições é mais provável o surgimento de rompimentos e discórdias na família, cuidados de saúde inadequados, desemprego frequiente, habitação inadequada, entre outros (Garmezy, 1991, 1993, 1996).

Uma colocação interessante em relação ao acúmulo de eventos de vida negativos e crônicos (por exemplo, discórdia entre os pais, criminalidade na família, desordem psiquiátrica da mãe, ausência de cuidados parentais) foi explicitada por Garmezy (1993) da seguinte forma, ao investigar fatores de risco familiares: a presença de um único evento estressor (ou nenhum estressor) produz aumento de um por cento nas chances de uma desordem psiquiátrica na criança. Dois estressores no meio familiar aumentam para cinco por cento a possibilidade da desordem; com três estressores, as chances crescem para seis por cento; com quatro ou mais eventos somam 21 por cento de chances da criança desenvolver uma desordem psiquiátrica. $\mathrm{O}$ acúmulo de estressores familiares potencializa 33 por cento da ocorrência de problemas psiquiátricos em crianças.

Aprofundando a idéia de cronicidade dos eventos, Rutter (1981) relembra que eventos agudos podem provocar efeitos negativos em curto prazo, mas nem sempre em longo prazo. Por exemplo, a hospitalização de uma criança por menos de uma semana geralmente não está associada com seqüelas posteriores. No entanto, pessoas expostas à adversidade crônica têm mais dificuldade em lidar com eventos de vida agudos. Por exemplo, o efeito de repetidas hospitalizações seria maior se associado a adversidades psicossociais do que em circunstâncias mais favoráveis de vida.

Os diferentes níveis individuais de tolerância ao estresse também oscilam conforme o período de vida em que o evento ocorre e conforme a situação enfrentada: uma pessoa é perturbada por pequenas mudanças, algumas são afetadas por eventos de maior magnitude; outras quando a exposição ao evento é mais prolongada ou ainda algumas podem alcançar seu limite de tolerância apenas com o acúmulo de pequenos eventos estressantes do dia-a-dia (Savoia, 1999).

Para Kaplan (1999), é a combinação entre a natureza, a quantidade e a intensidade dos fatores de risco o que define o contexto da adversidade necessária para a resiliência. Para Rutter (1987), pesquisador de referência para a área, a proximidade entre fatores de risco e proteção merece ser prioridade. Sugere que se use o termo risco sob a ótica de um mecanismo e não de um fator, uma vez que risco numa determinada situação pode ser proteção em outra. Alerta para o perigo em definir de forma arbitrária eventos isolados como fatores de risco, dando importância à idéia de equilíbrio entre risco e proteção, de forma que os primeiros sejam moderados pelos segundos, proporcionando como resultado uma atitude positiva frente à adversidade da vida.

Seja como for que se constitui o risco, é possível aprender formas de enfrentamento a partir da convivência com indivíduos que o vivenciaram e ultrapassaram com sucesso. A resposta do indivíduo ao risco tem sido descrita em termos de vulnerabilidade e resiliência. (Luthar, 1991; Antoni \& Koller, 2000). Por vulnerabilidade entende-se a predisposição individual para desenvolver variadas formas de psicopatologias ou comportamentos não eficazes, ou susceptibilidade para um resultado negativo no desenvolvimento. No outro lado, está a resiliência, como a predisposição individual para resistir às conseqüências negativas do risco e desenvolver-se adequadamente.

\section{Fatores protetores}

Pelos fatos explicitados anteriormente, constata-se que não é necessário compreender apenas a importância dos riscos para se aferir a resiliência. Os fatores ou mecanismos de proteção que um indivíduo dispõe internamente ou capta do meio em que vive são considerados elementos cruciais 
para a compreensão do tema. A etapa do desenvolvimento correspondente à infância e juventude tem sido priorizada pelos estudiosos do tema. Os termos mais utilizados para tratar a adversidade são fatores de risco, eventos de vida ou estressores, enquanto que os fatores de proteção, são, usualmente nomeados como mediadores (buffers).

Grande parte dos autores define de forma didática três tipos de fatores de proteção para a criança/adolescente (1) fatores individuais: auto-estima positiva, auto-controle, autonomia, características de temperamento afetuoso e flexível; (2) fatores familiares: coesão, estabilidade, respeito mútuo, apoio/suporte; (3) fatores relacionados ao apoio do meio ambiente: bom relacionamento com amigos, professores ou pessoas significativas que assumam papel de referência segura à criança e a faça sentir querida e amada (Brooks, 1994; Emery \& Forehand, 1996).

Gore e Eckenrode (1996) consideram que essa divisão tende a obscurecer as importantes formas de interrelação que ocorrem entre a pessoa e o meio. A eficácia do suporte social como redutor do estresse pode variar de acordo com o nível de outro fator de proteção como a auto-estima. A presença de um fator de proteção pode determinar o surgimento de outros fatores de proteção em algum outro momento. Portanto, compreender de que forma esses mediadores agem para atenuar os efeitos negativos do estresse ou do risco é tarefa tão complexa quanto determinar o que é fator de adversidade para cada ser humano.

Os processos de proteção têm a característica essencial de provocar uma modificação catalítica da resposta do indivíduo aos processos de risco. Possuem quatro principais funções: (1) reduzir o impacto dos riscos, fato que altera a exposição da pessoa à situação adversa; (2) reduzir as reações negativas em cadeia que seguem a exposição do indivíduo à situação de risco; (3) estabelecer e manter a auto-estima e auto-eficácia, através de estabelecimento de relações de apego seguras e o cumprimento de tarefas com sucesso; (4) criar oportunidades para reverter os efeitos do estresse (Rutter, 1987).

Há divergências na literatura sobre a capacidade desses fatores predizerem efetivamente a resiliência. Para Trombeta e Guzzo (2002), apenas os fatores de proteção são preditivos de resiliência, enquanto os fatores de risco não possuem tal capacidade. Para Yunes e Szymanski (2001), a resiliência é o produto final da combinação e acúmulo dos fatores de proteção.

Um conceito importante para se compreender a capacidade de resiliência do indivíduo é o de coping (Garmezy, 1988; Rutter, 1981, 1996). Refere-se ao posicionamento e às ações individuais frente às situações negativas de vida. São esforços cognitivos e comportamentais utilizados pelo indivíduo para lidar com as situações indutoras de estresse (Folkman \& Lazarus, 1985). Podem estar direcionados para a emoção (esforço para regular o estado emocional associado ao estresse) ou ser focalizado no problema (esforço para agir na origem do estresse, tentando modificá-lo). Estratégias de coping mais voltadas para o enfrentamento direto dos problemas ou a elaboração das dificuldades são mais encontradas em indivíduos resilientes, podendo moderar o efeito das adversidades, tornando-se um fator protetivo (Silva, 2001). Nos indivíduos resilientes há menor utilização de estratégias de coping de evitação dos problemas (Kavsek \& Seiffge-Krenke, 1996).

O coping ilustra a importância das diferenças individuais nos níveis social, psicológico e neuroquímico. A estratégia de coping utilizada pela criança ou adolescente não é baseada em um evento único, mas apreciada como resultado das experiências já vividas. O acúmulo de eventos ao longo do tempo é o que contribui para a resiliência ou vulnerabilidade psicológica. Por exemplo, Rutter $(1981,1989)$ questiona a possibilidade de experiências adversas nos primeiros anos de vida alterarem o curso de desenvolvimento subsequente ou influenciar nas respostas do indivíduo a eventos estressores que surjam em fases posteriores (juventude e vida adulta). O impacto de algum fator positivo ou negativo na infância, pode muitas vezes não ser manifestado a curto-prazo, mas contribuir na formação de cadeias de reações onde um evento negativo liga-se a outro ou por outro lado, um evento bemsucedido proporciona outros do mesmo tipo.

Diante dos fatores potencialmente geradores de desequilíbrio para cada indivíduo, os mecanismos de proteção são tomados como o ponto chave necessário para o restabelecimento do equilíbrio perdido e demonstração de competência apesar da adversidade. Confirmado que muitas pessoas escapam das conseqüências negativas do risco, foram intensificados os estudos focados em variáveis que podem operar nesse resultado positivo nas situações de vida.

Werner e Smith (2001), acompanhando indivíduos desde o nascimento até a idade de 40 anos, observaram claramente a relação entre fatores de risco e proteção. Entre as pessoas resilientes, quanto maior a quantidade de desvantagens e estresse acumulados ao longo da vida, mais fatores de proteção foram necessários durante a infância e juventude para contrabalançar os aspectos negativos de suas vidas e aumentar os resultados positivos no desenvolvimento. Destacaram como mediadores características de temperamento da criança e da sua família e suporte emocional dentro e fora da família. Os adultos resilientes eram descritos na primeira infância como pessoas mais afetuosas, ativas, de boa índole e fáceis de lidar. Quando adolescentes tenderam a mostrar maior auto-conceito, auto-controle, facilidade em interagir com amigos, professores e inserir-se em grupos.

Outro estudo longitudinal envolvendo crianças em situação de alto risco social observou que o desfecho positivo ou negativo na aquisição de competência deveu-se especialmente aos fatores de proteção (Rutter \& Quinton, 1984).

Conrad (1998), desenvolveu um estudo com crianças em situação de rua, filhas de mães com sintomas depressivos e surpreendeu-se ao verificar que 60 por cento dessas crianças não apresentaram em idades mais avançadas problemas de comportamento. Atribuiu esse resultado à presença de estruturas externas que oferecem segurança e suporte ao indivíduo.

O presente artigo é um ensaio exploratório sobre a associação da resiliência com eventos de vida adversos e com fatores de proteção em uma amostra de adolescentes escolares de uma rede pública de ensino. Apesar das diversas incertezas sobre os processos ocorridos no indivíduo até que consiga alcançar resiliência, acredita-se que as variáveis risco e proteção são peças fundamentais que se combinam no cenário complexo desse construto. 


\section{Método}

A amostra foi organizada por conglomerados, baseada na população dos alunos matriculados na $7^{\mathrm{a}} / 8^{\mathrm{a}}$ série do ensino fundamental e $1 \frac{a}{2} 2^{a}$ série do ensino médio de escolas públicas do município de São Gonçalo. Foram entrevistados 997 alunos entre 12 e 19 anos (idade média=15,4; $D P=1,48$ ), com supremacia de meninas $(57,7 \%)$ nas 17 escolas e 37 turmas que fizeram parte do estudo.

\section{Resiliência}

Neste estudo, a resiliência foi mensurada através da escala de Resiliência desenvolvida por Wagnild e Young (1993) que é um dos poucos instrumentos usados para medir níveis de adaptação psicossocial positiva frente a eventos de vida importantes. Possui 25 itens descritos de forma positiva com resposta tipo Likert variando de 1 (discordo totalmente) a 7 (concordo totalmente). Os escores da escala oscilam de 25 a 175 pontos, com valores altos indicando elevada resiliência. Estudos têm mostrado boa confiabilidade e validade desse instrumento (Wagnild \& Young; 1993), já adaptado para o português (Pesce, Assis, Avanci, Malaquias \& Oliveira, no prelo).

\section{Eventos de vida}

a) Família: um dos pais ou responsável já ficou ou está desempregado; problemas financeiros sérios na família; mora ou ter morado amontoado, sem espaço; familiar com problemas médicos sérios, como doenças ou acidentes; problema de deficiência física ou mental na família; familiar indiciado ou preso; morte de pai, mãe ou irmão; parente próximo morto; problemas de álcool ou drogas na família; discussões entre familiares envolvendo os filhos; família já se mudou muitas vezes de casa, bairro, cidade ou Estado; pais separados ou divorciados; pais se casaram novamente após separação; nascimento de um novo irmão ou irmã; morte de animal de estimação; ter ficado muito doente e recebido cuidados médicos.

b) Comunidade: viu alguém ser gravemente ferido; vive/ viveu em situação de perigo e insegurança na vizinhança; já teve a casa assaltada ou roubada.

Visando analisar se o número total de eventos de vida seria uma variável associada ao nível de resiliência foram criados: um indicador com o somatório de todos os eventos familiares relatados anteriormente e outro indicador com o somatório dos eventos ocorridos na comunidade. Cada um desses eventos de vida teve como opção de resposta sim ou não, com o valor correspondendo a 1 ou 0 , respectivamente.

\section{Violência como eventos de vida}

a) violência entre irmãos (alfa Cronbach 0.5 e índice de correlação intraclasse - ICC 0.85) e entre os pais (alfa 0.61 e ICC 0.71). Medido através da freqüência com que os irmãos/pais brigam a ponto de se machucarem ou humilham uns aos outros (muitas vezes, poucas vezes e nunca); b) violência física grave praticada por pai (alfa 0.83 e ICC 0.79 ) e mãe (alfa 0.79 e ICC 0.83 ) pelo menos uma vez na vida - Conflict Tatics Scales (Straus, 1979): chutar, morder ou dar murros, espancar, ameaçar ou efetivamente usar arma ou faca contra o adolescente. Apenas uma questão positiva indica presença de violência física grave. Foi adaptada para a língua portuguesa, apresentando bons resultados psicométricos (Assis, 1992; Hasselman, 1996). A violência grave da mãe e do pai se correlacionaram com a escala de auto-estima, violência psicológica e supervisão familiar, contribuindo para a validade de construto;

c) violência psicológica (alfa 0.91 e ICC 0.86) A escala de Pitzner e Drummond (1997) afere as experiências vividas pelo jovem, em que uma pessoa significativa denegriu suas qualidades, capacidades, desejos e emoções, além de cobrá-lo excessivamente. Correlacionou-se com a escala de apoio social, auto-estima, escalas que mensuram outras formas de violência, itens de eventos de vida e supervisão familiar. A soma dos escores de resposta (sempre, quase sempre, às vezes, raramente e nunca) foi categorizada de acordo com tercis, compondo níveis elevados, médios e baixos de violência psicológica;

d) violência sexual aferida pela questão "você teve experiências sexuais traumáticas quando era criança ou adolescente?", que teve como opção de resposta sim ou não.

\section{Fatores de proteção}

a) apoio social (Sherbourne \& Stewart conforme citado por Chor \& cols, 2001; Chor, Farstein, Alves \& Lopes, 2003): são apresentadas neste artigo quatro dimensões de apoio social: emocional (apoio recebido através da confiança, da disponibilidade em ouvir, compartilhar preocupações/medos e compreender seus problemas - ICC 0.83), de informação (através do recebimento de sugestões, bons conselhos, informação e conselhos desejados - ICC 0.82), afetiva (demonstração de afeto e amor, dar um abraço e amar - ICC 0.79) e de interação positiva (diversão juntos, relaxar, fazer coisas agradáveis e distrair a cabeça - ICC 0.77). Têm sido encontrados bons índices psicométricos na adaptação brasileira (Assis \& Avanci, 2004; Chor \& cols., 2001). Todas as dimensões desta escala se correlacionaram entre si e com a escala de violência psicológica. Os escores obtidos foram categorizados segundo tercil, configurando apoio social elevado, intermediário e fraco;

b) auto-estima (Rosenberg, 1989): 10 itens designados a avaliar globalmente a atitude positiva ou negativa de si mesmo (alfa de 0.76 e ICC de 0.81). Pode ser usada para adultos e adolescentes, obtendo uma alta consistência interna. Foi adaptado para o português, com bons índices psicométricos (Assis \& Avanci, 2004). Quanto à validade, a escala de auto-estima correlacionou-se com as escalas de violência, com os eventos de vida, com apoio emocional e interação positiva, o que teoricamente convergem com este construto. Os resultados foram categorizados, configurando auto-estima elevada, média e baixa;

c) supervisão familiar; medida através da frequiência com que os pais e responsáveis sabem onde o adolescente vai e com quem está; 
d) relacionamento com amigos e professores: opções de resposta: bom, regular e ruim.

O instrumento de coleta de dados foi organizado em cinco blocos: perfil sócio-demográfico do aluno, relacionamento com as pessoas do seu convívio (relações, supervisão familiar, escala de apoio social), o adolescente em relação a si mesmo e suas expectativas em relação ao futuro (escala de resiliência, escala de auto-estima, escala de satisfação de vida e itens sobre auto-confiança e auto-determinação); eventos negativos de vida; escalas de violência física, psicológica e sexual.

A formulação do questionário baseou-se em estudos e experiência dos pesquisadores (Fiocruz/Ensp/Claves, 2003; Minayo \& cols., 1999), deixando para a parte final temas mais delicados como eventos de vida e violência.

A pesquisa foi aprovada pelo Comitê de Ética em Pesquisa da Fundação Oswaldo Cruz.

\section{Análise}

Foram utilizados o Teste $t$ e a Análise de Variância (ANOVA) para se encontrar diferenças significativas ao nível de $5 \%$ nas variáveis quantitativas e qualitativas ordinais. $\mathrm{O}$ Teste $t$ foi empregado nas variáveis qualitativas nominais binárias, para testar a diferença da variável quantitativa resiliência com as médias dos grupos das variáveis dicotômicas. A análise de variância (ANOVA) foi usada para testar a diferença com as variáveis qualitativas ordinais com mais de duas categorias; o teste de Tukey efetuou a comparação múltipla das médias dos grupos.

\section{Resultados}

Na Tabela 1 pode se verificar a inexistência de associação da resiliência com todos os eventos adversos de vida investigados, sejam eles oriundos ou não de violência. Também não se verifica distinção quantitativa entre o total de eventos de vida vivenciados pelos jovens na família e comunidade e o nível de resiliência que alcançam. A variável sexo foi a única associada a resiliência, com as meninas apresentando níveis maiores de superação das dificuldades que os meninos.

A análise dos eventos adversos de vida segundo o sexo constatou que apenas a violência sexual mostrou associação com resiliência nos rapazes, com média de 71,7 entre os que nunca sofreram e 65,4 entre os que sofreram ( $p=0,03$, dados não apresentados).

Em relação aos fatores protetores, verifica-se na Tabela 2, uma situação diferenciada. Adolescentes com maiores níveis de resiliência têm: auto-estima mais elevada; maior supervisão familiar mensurada através da frequiência em que os pais sabem aonde vai o adolescente quando sai de casa e com quem está; melhor relacionamento com outras pessoas como amigos e professores; maior apoio social, seja ele emocional, afetivo, informacional e de interação positiva.

Constatou-se também que adolescentes mais resilientes tendem a ter sempre um adulto em casa supervisionando-os na volta da escola (dados não apresentados).

Para exemplificar a análise da homogeneidade das respostas para cada variável presente na Tabela 2, observa-se que as médias dos três níveis de auto-estima diferem uma das
Tabela 1. Resiliência segundo variáveis sócio-demográficas e eventos adversos de vida

\begin{tabular}{|c|c|c|c|c|}
\hline \multicolumn{2}{|l|}{ Variáveis } & \multirow{2}{*}{$\frac{\mathbf{N}}{352}$} & \multirow{2}{*}{$\begin{array}{l}\text { Média } \\
73,54\end{array}$} & \multirow{2}{*}{$\frac{p \text {-valor }}{0,008}$} \\
\hline Sexo* & Feminino & & & \\
\hline & Masculino & 266 & 71,34 & \\
\hline \multirow[t]{2}{*}{ faixa etária* } & $10-14$ anos & 175 & 72,48 & ns \\
\hline & $15-19$ anos & 444 & 72,64 & \\
\hline \multirow[t]{3}{*}{ Violência psicológica** } & Baixa & 154 & 74,12 & ns \\
\hline & Média & 173 & 72,17 & \\
\hline & Alta & 163 & 72,14 & \\
\hline \multirow[t]{2}{*}{ Violência física severa - pai* } & Não & 452 & 72,78 & ns \\
\hline & Sim & 91 & 72,30 & \\
\hline \multirow[t]{2}{*}{ Violência física severa - mãe* } & Não & 426 & 72,88 & ns \\
\hline & Sim & 179 & 72,11 & \\
\hline \multirow[t]{3}{*}{$\begin{array}{l}\text { Violência física entre } \\
\text { irmãos** }\end{array}$} & Nunca & 37 & 71,06 & ns \\
\hline & Muitas Vezes & 336 & 72,71 & \\
\hline & Poucas Vezes & 157 & 72,86 & \\
\hline \multirow[t]{3}{*}{ Violência física entre pais** } & Nunca & 8 & 67,86 & ns \\
\hline & Poucas Vezes & 83 & 71,37 & \\
\hline & Muitas Vezes & 420 & 73,00 & \\
\hline \multirow[t]{2}{*}{ Violência sexual* } & Não & 585 & 72,85 & ns \\
\hline & Sim & 28 & 70,14 & \\
\hline \multirow[t]{3}{*}{ eventos: família** (0-16) } & $0-1$ & 4 & 68,43 & ns \\
\hline & $2-3$ & 39 & 75,12 & \\
\hline & $4 \mathrm{ou}+$ & 529 & 72,69 & \\
\hline \multirow[t]{2}{*}{ eventos: comunidade* $(0-3)$} & $0-1$ & 448 & 72,98 & ns \\
\hline & $2-3$ & 168 & 71,44 & \\
\hline \multirow[t]{3}{*}{$\begin{array}{l}\text { Total de eventos de vida**: } \\
(0-27)\end{array}$} & $0-7$ & 219 & 73,02 & \\
\hline & $6-13$ & 340 & 72,21 & ns \\
\hline & 14 ou mais & 55 & 73,44 & \\
\hline
\end{tabular}

* Teste $t$; ** ANOVA; ns: não significativo

outras, com um nítido gradiente de redução da auto-estima à medida que decai o nível de resiliência. Tomando como exemplo o relacionamento com professores, alunos que respondem ter relacionamento bom ou regular têm maior nível de resiliência, distinguindo-se dos que respondem ter relacionamento ruim.

Outras variáveis mostraram uma gradação diferenciada: a maioria das diferenças de médias foi observada para as variáveis extremas (apoio elevado versus fraco ou relacionamento bom versus ruim), com o nível intermediário associando-se a um dos pólos de resposta.

\section{Discussão}

Os dados encontrados neste estudo mostraram que os eventos traumáticos de vida não estão relacionados com características de um indivíduo resiliente. Não se pode afirmar que ter vivenciado um tipo de evento ou várias experiências negativas contribui para a capacidade de lidar com a adversidade. Esses achados, ainda preliminares, estão de acordo com a teoria que não encontra relação direta entre risco e resiliência, pois há que se considerar o processamento da experiência negativa como fator importante para a resiliência. Portanto, 


\section{R. P. Pesce e cols.}

Tabela 2. Resiliência segundo fatores protetores individuais, familiares e de apoio/suporte do meio ambiente

\begin{tabular}{|c|c|c|c|c|c|}
\hline \multicolumn{2}{|c|}{ Variáveis } & \multirow{2}{*}{$\frac{\mathbf{N}}{191}$} & \multirow{2}{*}{$\begin{array}{l}\text { Média } \\
76,5056^{\mathrm{a}}\end{array}$} & \multirow{2}{*}{$\begin{array}{c}\mathbf{F} \\
\mathrm{F}_{2,595}=23,902\end{array}$} & \multirow{2}{*}{$\begin{array}{r}p \text { valor } \\
0,000\end{array}$} \\
\hline Auto-estima & Alta & & & & \\
\hline & Média & 167 & $72,3285^{b}$ & & \\
\hline & Baixa & 240 & $69,8976^{\mathrm{c}}$ & & \\
\hline \multirow[t]{3}{*}{ Supervisão familiar } & Sempre & 443 & 73,1945 & $\mathrm{~F}_{2,614}=3,046$ & 0,048 \\
\hline & Muitas Vezes & 105 & 71,8476 & & \\
\hline & Poucas vezes ou nunca & 69 & 70,1284 & & \\
\hline \multirow[t]{3}{*}{ Relacionamento - amigos } & Bom & 562 & 73,0869 & $\mathrm{~F}_{3,615}=13,309$ & 0,001 \\
\hline & Regular & 51 & 67,8768 & & \\
\hline & Ruim e sem amigos & 6 & 66,5714 & & \\
\hline \multirow[t]{3}{*}{ Relacionamento - professores } & Bom & 473 & $73,2504^{\mathrm{a}}$ & $F_{2,616}=6,065$ & 0,000 \\
\hline & Regular & 137 & $70,8905^{\mathrm{a}}$ & & \\
\hline & Ruim & 9 & $64,0635^{\mathrm{b}}$ & & \\
\hline \multirow[t]{3}{*}{ Apoio afetivo } & Elevado & 204 & $69,9832^{\mathrm{a}}$ & $\mathrm{F}_{2,602}=15,480$ & 0,000 \\
\hline & Intermediário & 160 & $72,4393^{b}$ & & \\
\hline & Fraco & 244 & $75,1522^{\mathrm{c}}$ & & \\
\hline \multirow[t]{3}{*}{ Apoio emocional } & Elevado & 230 & $70,1839^{\mathrm{a}}$ & $\mathrm{F}_{2,598}=14,734$ & 0,000 \\
\hline & Intermediário & 199 & $73,5678^{\mathrm{a}}$ & & \\
\hline & Fraco & 172 & $75,2392^{b}$ & & \\
\hline \multirow[t]{3}{*}{ Apoio de informação } & Elevado & 231 & $70,2041^{a}$ & $\mathrm{~F}_{2,598}=20,198$ & 0,000 \\
\hline & Intermediário & 184 & $72,382^{\mathrm{a}}$ & & \\
\hline & Fraco & 186 & $76,2427^{b}$ & & \\
\hline \multirow[t]{3}{*}{ Interação positiva } & Elevado & 240 & $70,119^{a}$ & $\mathrm{~F}_{2,597}=16,488$ & 0,000 \\
\hline & Intermediário & 168 & $73,4592^{b}$ & & \\
\hline & Fraco & 192 & $75,4375^{\mathrm{b}}$ & & \\
\hline
\end{tabular}

a, b, c - para cada variável que foi possível executar o Teste de Tukey, a mesma letra indica homogeneidade da média. Quando são usadas as letras a, b, c, numa mesma variável, constata-se que as três médias são diferentes entre si.

a hipótese não deveria ser que uma vivência de violência ou qualquer outro evento adverso causa necessariamente prejuízos na vida do adolescente, mas sim que quando o jovem sucumbe ao risco, ele está propenso a ter mais dificuldade em sair-se bem frente aos desafios da vida. Isso não significa desprezar os efeitos maléficos de, por exemplo, uma violência grave contra o jovem, mas pelo contrário enfatizar que é possível "sobreviver" a essa situação, ainda que sempre seja inaceitável que aconteça.

Os fatores de proteção, por sua vez, tiveram associação com a resiliência, confirmando a hipótese teórica que molda esse construto. Pode-se interpretar essa relação entendendo que os fatores de proteção aqui abordados (auto-estima, apoio social, gênero, relacionamento com outras pessoas) atuam como facilitadores no processo individual de perceber e enfrentar o risco.

Os resultados aqui expostos mostram ainda que focar um evento de vida isoladamente atribuindo-lhe adversidade ou considerar o somatório destes como a condição de risco não parece ser a melhor forma de abordar a questão. É imprescindível uma análise mais criteriosa dos processos que de fato proporcionam adversidade à vida. Kaplan (1999) enfatiza que não existe um critério que afirme que uma variável particular determine um fator de risco ou fator de proteção, ou ainda que ambos estejam relacionados com um resultado resiliente. Definir risco e proteção é um problema trivial exceto pelo fato de que definir maior ou menor vulnerabilidade depende de uma determinação inicial do que é considerado risco.
Para definir um fator que protege uma criança em risco de um resultado pouco resiliente, é preciso definir o significado de risco em algum lugar entre os dois pólos: a situação ou circunstância que oferece risco e o resultado mais ou menos resiliente. Nesse meio do caminho podem estar os fatores que oferecem proteção e que vão de alguma forma, ajudar o indivíduo a dimensionar o risco.

Os adolescentes investigados nesse estudo pertencem a uma classe sócio-econômica desfavorecida, fato que pode ser interpretado como risco devido às desvantagens associadas e que pode ter contribuído de alguma forma para os resultados observados no presente estudo. Garmezy (1991) afirma que dos atributos positivos observados em crianças que vivem em meio a pobreza, alguns são instilados pela família, alguns pela escola e alguns por outras instituições como a igreja. Outros são inerentes ao indivíduo e contribuem para a força necessária aos jovens que sofrem com a adversidade biológica, física, social ou cognitiva. O poder de um fator de proteção, segundo o autor, pode amortecer a tendência antissocial, a auto-estima perdida e a performance e competência sem brilho, que tende a ser conseqüência de elementos de risco presentes em um ambiente marcado pela desvantagem e privação.

A utilização de referencial estatístico mais elaborado poderia propiciar o aprofundamento do conhecimento, ainda em fase inicial de elaboração. A análise dos efeitos da interação entre risco e proteção deve ser o próximo caminho, buscando-se observar os efeitos dessa combinação, levando-se em 
conta a precocidade de se investigar esses atributos ainda na adolescência, tendo por princípio as alterações vivenciadas de acordo com o momento cronológico, a natureza das experiências e a percepção individual.

A opção de utilizar variáveis de natureza diferente foi definida pela relevância dos fatores de risco e proteção na fundamentação teórica sobre resiliência. É comum em estudos sobre resiliência, a utilização de variados instrumentos de natureza e tamanhos diferentes principalmente pela dificuldade em se atingir a resiliência através de um único parâmetro de medida. Por esse motivo, a escala de resiliência foi comparada com variáveis afins como os eventos negativos de vida (fundamentais para existência da resiliência), a auto-estima e outras características de personalidade (que teoricamente estariam positivamente correlacionadas com a resiliência) e apoio social (também uma das bases para constituição de uma personalidade fortificada).

Mais do que problematizar a agregação de diferentes variáveis, importa qualificar as propriedades psicométricas de cada escala utilizada. No presente estudo, os resultados da validade de construto dão força ao recorte utilizado (Pesce $\&$ cols., no prelo).

Luthar (1991) utilizou de forma similar diversas medidas para alcançar resiliência em adolescentes de escolas públicas. Os instrumentos escolhidos mensuravam eventos de vida, características demográficas da população, medidas de competência na escola e entre colegas, características individuais de personalidade como inteligência, comportamentos sociais e auto-controle, além de medidas para sintomatologia de depressão e ansiedade.

Sem dúvida o conceito de resiliência tem sido fundamental no campo do desenvolvimento e da saúde humana. Os conceitos de risco e proteção não podem ser considerados homogêneos. É necessária uma análise discriminada apontando para a possibilidade de diferentes grupos de eventos de vida adversos proporcionarem efeitos de qualidades distintas na vida do indivíduo. Da mesma forma, os fatores de proteção, apesar de terem mostrado uma relação mais direta com a resiliência, devem ser entendidos tanto separadamente quanto em conjunção com outros fatores e com o próprio risco.

Outra limitação do trabalho refere-se à própria definição de resiliência, pois ainda "não possui uma definição única, nem um parâmetro inquestionável, nem uma medida unifor$m e "$ (Slap, 2001, p. 173).

Diante dessas questões, é importante destacar como os fatores de proteção atuam sobre os eventos de vida adversos e em que ocasião um evento (ou eventos) apresenta-se como ameaça ao desenvolvimento da criança e do adolescente.

Definir com segurança o que constitui ou não um fator de proteção ou um fator de risco exige cuidados, principalmente quando se pensa nas crianças e adolescentes que são privados de uma base familiar sólida e de acesso a rede social de apoio e ainda assim se mostram resilientes e competentes frente à adversidade. Para esse indivíduo sobraria apenas a lista de qualidades pessoais. Se a resiliência for vista nessa perspectiva individual torna-se complicada a elaboração de programas ou intervenção social, fato que vem sendo alvo de muitos estudos sobre o tema (Bloom, 1996; Masten, 1999; Munist \& cols., 1998).

\section{Considerações Finais}

A emergência dos estudos em resiliência principalmente nas últimas duas décadas representa uma abordagem para conhecer como a criança se desenvolve quando confrontada com circunstâncias adversas. Nestas situações, muito se focalizava nos fatores de risco e na etiologia dos problemas da criança e adolescente (como uso de álcool e drogas, psicopatologia, delinqüência e comportamento violento). No entanto, importantes aprendizados podem ser adquiridos pela convivência com pessoas que ultrapassaram com sucesso o risco. A investida na resiliência tem enfraquecido o foco na "patologia da desvantagem" e valorizado aqueles que obtêm sucesso de alguma forma em suas vidas.

Este estudo foi uma tentativa de contribuir para a compreensão desse constructo, que embora estimulante, deixa antever muitas questões a serem abordadas no futuro. Um fator associado à resiliência em um domínio pode ser efetivo em outros domínios? De que forma os processos relacionados à resiliência mudam com o passar do tempo? Pode-se falar sobre resiliência da mesma forma em estudos relacionados à resistência ao estresse e àqueles que envolvem boa recuperação ao trauma? (Al-Naser \& Sandman, 2000; Jenkins, 1997).

Mesmo sabendo-se dos avanços que a resiliência pode oferecer na área da saúde, deve-se ter a preocupação em não transformar a resiliência num conceito "da moda" que subestime circunstâncias de vida penosas para a criança, como, por exemplo, o contexto de violência. A ênfase na promoção da resiliência não deve substituir as políticas de combate à desigualdade social e condições de vida precárias de alguns sujeitos. A resiliência, incorporada na medida certa aos estudos nessa área, pode render frutos louváveis.

\section{Referências}

Al-Naser, F. \& Sandman, M. (2000). Evaluating resilience factors in the face of traumatic events in Kuwait. Medical Sciences, 2, 111-116.

Antoni, C. de \& Koller, S. H. (2000). Vulnerabilidade e resiliência familiar: Um estudo com adolescentes que sofreram maus tratos intrafamiliares. Psico, 31(1), 39-66.

Assis, S. G. (1992). Violência, criança e comportamento: Um estudo em grupos sociais distintos. Relatório final de pesquisa. Rio de Janeiro: Fundação Oswaldo Cruz/ Escola Nacional de Saúde Pública/ Centro Latino-Americano de Estudos sobre Violência e Saúde - Jorge Careli.

Assis, S. G. \& Avanci, J. Q. (2004). Labirinto de espelhos. Formação da auto-estima na infância e na adolescência. Rio de Janeiro: Editora Fiocruz.

Bloom, M. (1996). Primary prevention and resilience: changing paradigms and changing lives. Em R. L Hampton, P. Jenkins \& T. P. Gulotta (Orgs.), Preventing Violence in America (pp. 87-114). California: Sage.

Brooks, R. (1994). Children at risk: fostering resilience and hope. American Journal of Orthopsychiatry, 64(4), 545-553.

Celia, S. \& Souza, R. P. (1999). Risco e resiliência. Em M. C. O. Costa \& R. P. de Souza (Orgs.), Adolescência: Aspectos clínicos e psicossociais (pp. 333-339). Porto Alegre: Artmed. 
Chor, D., Grip, R. H., Lopes, C. S. \& Farstein, E. (2001). Medidas de rede e apoio social no Estudo Pró-Saúde: pré-testes e estudo piloto. Cadernos de Saúde Pública, 17(4), 887-896.

Chor, D., Farstein E., Alves, M. G. M. \& Lopes, C. de S. (2003). How reproducible is self-reported information on exposure to smoking, drinking and dietary patters? Evidence among brazilian adults in the pró-saúde study. São Paulo Medical Journal, 12(2), 63-66.

Conrad, B. S. (1998). Maternal depressive symptoms and homeless children's mental health: Risk and resiliency. Archives of Psychiatric Nursing, 12(1), 50-58.

Engle, P. L., Castle, S. \& Menon, P. (1996). Child development: Vulnerability and resilience. Social Science Medicine, 43(5), 621-635.

Emery, R. E. \& Forehand, R. (1996). Parental divorce and children's well-being: A focus on resilience. Em R. J. Haggerty, L. R. Sherrod, N. Garmezy \& M. Rutter (Orgs.), Stress, risk and resilience in children and adolescents. Processes, mechanisms and interventions (pp. 64-100). Cambridge: Cambridge University Press.

Folkman, S. \& Lazarus, R. S. (1985). If it changes it must be a process: Study of emotion and coping during three stages of a college examination. Journal of personality and social psychology, 48(1), 150-170.

FIOCRUZ/ENSP/CLAVES (2003). Avaliação do processo de implantação e dos resultados do programa cuidar. Retirado do www.institutosouzacruz.org.br

Garmezy, N.(1988). Stressors of childhood. Em N. Garmezy \& M. Rutter (Orgs.), Stress, coping and development in children (pp. 43-105). New York: Johns Hopkins University Press.

Garmezy, N. (1991). Resiliency and vulnerability to adverse developmental outcomes associated with poverty. American Behavioral Scientist, 34(4), 416-430.

Garmezy, N. (1993). Children in poverty: resilience despite risk. Psychiatry, 56, 127-136.

Garmezy, N. (1996). Reflections and commentary on risk, resilience and development. Em R. J. Haggerty, L. R. Sherrod, N. Garmezy $\&$ M. Rutter (Orgs.), Stress, risk and resilience in children and adolecents: processes, mechanisms and interventions (pp. 1-19). Cambridge: Cambridge University Press.

Gore, S. \& Eckenrode, J. (1996). Context and process in research on risk, resilience and development. Em R. J. Haggerty, L. R. Sherrod, N. Garmezy \& M. Rutter (Orgs.), Stress, risk and resilience in children and adolecents: processes, mechanisms and interventions (pp. 19-64). Cambridge: Cambridge University Press.

Hasselmann, M. H. (1996). Violência familiar e desnutrição severa na infância: Modelo teórico e estudo de confiabilidade dos instrumentos. Dissertação de Mestrado, Universidade Estadual do Rio de Janeiro, Rio de Janeiro.

Jenkins, J. H. (1997). Not without a trace: Resilience and remembering among Bosnian refugees (commentary on "A family survives genocide"). Psychiatry, 60(1), 40-43.

Junqueira, M. F. P. \& Deslandes, S. F. (2003). Resiliência e maus tratos à criança. Cadernos de Saúde Pública, 19(1), 227-335.

Kaplan, H. B. (1999). Toward an understanding of resilience: A critical review of definitions and models. Em M. D. Glantz \& J. L. Johnson (Orgs.), Resilience and development. Positive life adaptations (pp. 17-83). New York: Plenum Press.
Kavsek, M. J, \& Seiffge-Krenke, I. (1996). The differentiation of coping traits in adolescence. International Journal of Behavioral Development, 19(3), 651-668.

Luthar, S. S. (1991). Vulnerability and resilience: A study of highrisk adolescents. Child Development, 62, 600-616.

Luthar, S. S. \& Cushing, G. (1999). Measurement issues in the empirical study of resilience: An overview. Em M. D. Glantz \& J. L. Johnson (Orgs.), Resilience and development: Positive life adaptations (pp. 129-160). New York: Plenum Press.

Luthar, S. S. \& Zigler, E. (1991). Vulnerability and competence: A review of reaserch on resilience in childhood. American Orthopsychiatric Association, 61(1), 6-23.

Masten, A. S. (1999). Commentary: The promise and perils of resilience research as a guide to preventive interventions. Em M. D. Glantz \& J. L. Johnson (Orgs.), Resilience and development. Positive life adaptations (pp. 251-259). New York: Plenum Press.

Minayo, M. C. S., Assis, S. G., Souza, E. R., Njaine, K., Deslandes, S. F., Silva, C. M. F., Fraga, P. C. P., Gomes, R., Abramovay, M., Waiselfisz, J. J. \& Monteiro, M. C. (1999). Fala galera. Juventude, violência e cidadania na cidade do Rio de Janeiro. Rio de Janeiro: Garamond.

Munist, M., Santos, H., Kotliarenco, M. A., Ojeda, E. N., Infante, F. \& Grotberg, E. (1998). Manual de identificación y promoción de la resiliencia. Washington: OPAS.

Pesce, R., Assis, S. G., Avanci, J., Malaquias, J. \& Oliveira, R. V. C. (no prelo). Adaptação transcultural, confiabilidade e validade da escala de resiliência Cross-Cultural adaptation, Reliability and Validity of the Resilience Scale. Cadernos de Saúde Pública.

Pitzner, J. K. \& Drummond, P. D. (1997). The reliability and validity of empirically scaled measures of psychological/verbal control and physical/sexual abuse: Relationship between current negative mood and a history of abuse independent of other negative life events. Journal of Psychosomatic Research, 43(2), 125-142.

Rosenberg, M. (1989). Society and the adolescent self-image. Princeton, NJ: Princeton University Press.

Rutter, M. (1981). Stress, coping and development: Some issues and some questions. Journal of Child Psychology and Psychiatry, 22(4), 323-356.

Rutter, M. (1987). Psychosocial resilience and protective mechanisms. American Orthopsychiatric Association, 57(3), 316-331.

Rutter, M. (1989). Pathways from childhood to adult life. Journal of Child Psychology and Psychiatry, 30(1), 23-51.

Rutter, M. (1996). Stress research: Accomplishments and tasks ahead. Em R. J. Haggerty, L. R. Sherrod, N. Garmezy \& M. Rutter (Orgs.), Stress, risk and resilience in children and adolecents: processes, mechanisms and interventions (pp. 354387). Cambridge: Cambridge University Press.

Rutter, M. \& Quinton, D. (1984). Long-term follow-up of women institutionalized in childhood: Factors promoting good functioning in adult life. British Journal of Developmental Psychology, 18, 225-234.

Savoia, M. G. (1999). Escalas de eventos vitais e de estratégias de enfrentamento (coping). Revista de Psiquiatria Clínica, 26(2), 57-67.

Slap, G. B. (2001). Conceitos atuais, aplicações práticas e resiliência no novo milênio. Adolescência Latino Americana, 2(3), 173-176. 
Silva, D. F. M. (2001). O desenvolvimento das trajetórias do comportamento delinqüente em adolescentes infratores. Tese de Doutorado, Universidade Federal do Rio Grande do Sul, Porto Alegre.

Straus, M. A. (1979). Measuring intrafamiliar conflict and violence. The Conflict Tactics (CT) Scales. Journal of Marriage and the Family, 41, 75-88.

Trombeta, L. H. A. \& Guzzo, R. S. (2002). Enfrentando o cotidiano adverso. Estudo sobre resiliência em escolares. Campinas: Alínea.

Yunes, M. A. M. \& Szymanski, H. (2001). Resiliência: noção, conceitos afins e considerações críticas. Em J. Tavares (Org.), Resiliência e educação (pp. 13-42). São Paulo: Cortez.
Wagnild, G. M. \& Young, H. M. (1993). Development and psychometric evaluation of resilience scale. Journal of Nursing Measurement, 1(2), 165-178.

Werner, E. \& Smith, R. S. (2001). Journeys from childhood to midlife. Risk, resilience and recovery. Ithaca: Cornell University Press.
Recebido em 04.02.2004

Primeira decisão editorial em 07.06.2004

Versão final em 08.07.2004

Aceito em 21.07.2004

\section{INTERESSADO EM AGILIZAR A PUBLICAÇÃO DE SEU ARTIGO?}

\section{Informações e dicas para os autores sobre aspectos operacionais}

\section{O manuscrito é reencaminhado pelo autor em versão reformulada}

Quando a versão reformulada é aceita e são poucos os reparos, as correções necessárias são feitas na secretaria da revista. Com o disquete disponível, o processamento pode ser imediatamente iniciado. Na falta de disquete ou CD-R, não é possível o processamento de correções nem a preparação do manuscrito para editoração. Em suma, atraso para entrar no prelo.

\section{Informações e dicas para os autores com artigos no prelo}

\section{O manuscrito é encaminhado para editoração}

No caso de o manuscrito ser aceito para publicação, é esperada a participação do autor no processo de preparação do artigo na fase de editoração. A primeira prova do artigo, editada a partir da versão em disquete ou CD-R, é enviada para exame ao(s) autor(es). O prazo para devolução é de 48 horas. A obediência ao prazo permite a manutenção da composição prevista para $\mathrm{o}$ volume/número da revista. $\mathrm{O}$ atraso no envio da prova examinada pode implicar em mudanças na composição de um número no prelo. E pode resultar em atraso na edição final. 